T. MURAI

KODAI MATH. J.

5 (1982), 313-317

\title{
THE VALUE-DISTRIBUTION OF RANDOM ENTIRE FUNCTIONS
}

\author{
By TAKAFUMi MURAI
}

1. It is well-known that, for a given entire function $f(z), \delta(a, f)=0 \quad(a \in \boldsymbol{C})$ holds except possibly for a countable set, where " $\delta$ " denotes the deficiency and $C$ the complex plane. We cannot generally remove the above exceptional set. The purpose of this paper is to show that the totality of entire functions $f(z)$ with $\delta^{*}(f)=\sup _{a \in C} \delta(a, f)>0$ is thin in a sense.

An open interval $\Omega=(-1 / 2,1 / 2)$ is naturally a probability space. A Rademacher series $\varepsilon=\left(\varepsilon_{k}\right)_{k=1}^{\infty}$ in $\Omega$ is defined by $\varepsilon_{k}(\omega)=\operatorname{sign}\left(\sin 2^{k} \pi \omega\right)(\omega \in \Omega)$. For a sequence $\left(a_{k}\right)_{k=1}^{\infty}(\not \equiv 0) \subset C$ with $\limsup _{k \rightarrow \infty}\left|a_{k}\right|^{1 / k}=0$, a random entire function is defined by

$$
f_{\varepsilon}(z)=\sum_{k=1}^{\infty} \varepsilon_{k} a_{k} z^{k}=\left\{f_{\omega}(z)=\sum_{k=1}^{\infty} \varepsilon_{k}(\omega) a_{k} z^{k} ; \omega \in \Omega\right\} .
$$

A random entire function $f_{\varepsilon}(z)$ is a probability space of entire functions. We write simply $\delta(a, \omega)=\delta\left(a, f_{\omega}\right), \delta^{*}(\omega)=\delta^{*}\left(f_{\omega}\right)$. In this paper, we shall show the following

THEOREM. $\quad \delta^{*}(\omega)=0$ almost surely (a.s.).

2. We denote by "Pr" the probability. Put

$$
\left\{\begin{array}{l}
T\left(r, f_{\omega}\right)=1 / 2 \pi \int_{0}^{2 \pi} \log ^{+}\left|f_{\omega}\left(r e^{i t}\right)\right| d t \\
T_{0}(r)=\log ^{+} A_{0}(r), \quad A_{0}(r)=\left(\sum_{k=1}^{\infty}\left|a_{k}\right|^{2} r^{2 k}\right)^{1 / 2} \\
m(r, a, \omega)=1 / 2 \pi \int_{0}^{2 \pi} \log ^{+} 1 /\left|f_{\omega}\left(r e^{i t}\right)-a\right| d t \quad(a \in C, r>0),
\end{array}\right.
$$

where $\log ^{+} x=\max \{\log x, 0\}(x>0)$. Note that $\delta(a, \omega)=\liminf _{r \rightarrow \infty} m(r, a, \omega) / T\left(r, f_{\omega}\right)$ $(a \in C, \omega \in \Omega)$. If $\#\left\{k ; a_{k} \neq 0\right\}<\infty$, then $f_{\varepsilon}(z)$ is a probability space of polynomials and we see easily $\delta^{*}(\omega)=0$ for all $\omega \in \Omega$. The proof in the case where

Received March 20, 1981 
$\#\left\{k ; a_{k} \neq 0\right\}=\infty$ is essential. For the sake of simplicity, we only give the proof in the case where $a_{k} \neq 0$ for all $k$. We use the following proposition, which is an improvement of Lemma 4 in [5].

Proposition. Suppose that there exist a set $\Omega_{0} \subset \Omega$ with $\operatorname{Pr}\left(\Omega_{0}\right)=1$ and mappings $\delta(\cdot ; m, q, p)(p=1, \cdots, q ; q=1,2, \cdots ; m=1,2, \cdots)$ from $\Omega_{0}$ to an interval $[0,1]$ such that:

(3) If $\omega \in \Omega_{0}$ and $\omega^{\prime}$ satısfy $\varepsilon_{k}\left(\omega^{\prime}\right)=\varepsilon_{k}(\omega)$ except for a. finite number of $k^{\prime}$, then $\omega^{\prime} \in \Omega_{0}$.

(4) $\delta^{*}(\omega) \leqq \delta(\omega ; m, q, p)$.

(5) $\sum_{\omega^{\prime} \in \Gamma(\omega, m, p)} \delta\left(\omega^{\prime} ; m, q, p\right) \leqq 1$ for all $\omega \in \Omega_{0}$, where $\Gamma(\omega, m, p)=\left\{\omega^{\prime} \in \Omega_{0}\right.$; $\varepsilon_{k}\left(\omega^{\prime}\right)=\varepsilon_{k}(\omega)$ for all $k$ with $\left.k \neq(p-1) m+1,(p-1) m+2, \cdots, p m\right\}$.

(6) $\delta(\omega ; m, q, p)=\delta\left(\omega^{\prime} ; m, q\right.$, p) if $\varepsilon_{k}(\omega)=\varepsilon_{k}\left(\omega^{\prime}\right)$ for all $k \geqq(p-1) m+1$.

Then $\delta^{*}(\omega)=0$ a.s..

Proof. For a sequence $\left(\varepsilon_{n}, \varepsilon_{n+1}, \cdots\right), \varepsilon_{k}= \pm 1$, we put $\Omega\left(\varepsilon_{n}, \varepsilon_{n+1}, \cdots\right)=$ $\left\{\omega \in \Omega_{0} ; \varepsilon_{k}(\omega)=\varepsilon_{k}(k \geqq n)\right\}$. Let $m, q \geqq 1$ and $\left(\alpha_{q m+1}, \alpha_{q m+2}, \cdots\right), \alpha_{k}= \pm 1$, be a sequence such that $\Omega\left(\alpha_{q m+1}, \alpha_{q m+2}, \cdots\right) \neq \Phi$. Then, by $(3), \sharp \Omega\left(\alpha_{q m+1}, \cdots\right)=2^{q m}$. Now we show that there exists an $m$-tuple $\left(\beta_{(q-1) m+1}, \cdots, \beta_{q m}\right), \beta_{k}= \pm 1$, such that $\delta^{*}(\omega) \leqq 2^{-m}\left(\omega \in \Omega\left(\beta_{(q-1) m+1}, \cdots, \beta_{q m}, \alpha_{q m+1}, \alpha_{q m+2}, \cdots\right)\right)$.

To see this, choose arbitrarily $\omega_{0} \in \Omega\left(\alpha_{q m+1}, \cdots\right)$. By (5), we have

$$
\sum_{\omega \in \Gamma\left(\omega_{0}, m, q\right)} \delta(\omega ; m, q, q) \leqq 1 .
$$

Since $\sharp \Gamma\left(\omega_{0}, m, q\right)=2^{m}$, there exists $\omega_{0}^{\prime}$ such that $\delta\left(\omega_{0}^{\prime} ; m, q, q\right) \leqq 2^{-m}$. Put $\beta_{k}=$ $\varepsilon_{k}\left(\omega_{0}^{\prime}\right) \quad(k=(q-1) m+1, \cdots, q m) . \quad$ Then $\delta^{*}(\omega) \leqq \delta(\omega ; m, q, q)=\delta\left(\omega_{0}^{\prime} ; m, q, q\right) \leqq 2^{-m}$ $\left(\omega \in \Omega\left(\beta_{(q-1) m+1}, \cdots, \beta_{q m}, \alpha_{q m+1}, \cdots\right)\right)$. Thus the required $m$-tuple $\left(\beta_{(q-1) m+1}, \cdots, \beta_{q m}\right)$ is obtained.

The above fact signifies that $\delta^{*}(\omega) \leqq 2^{-m}\left(\omega \in \Omega\left(\varepsilon_{(q-2) m+1}, \cdots, \varepsilon_{q m}, \alpha_{q m+1}, \cdots\right)\right)$ holds if $\left(\varepsilon_{(q-1) m+1}, \cdots, \varepsilon_{q m}\right)=\left(\beta_{(q-1) m+1}, \cdots, \beta_{q m}\right)$, and hence it holds for the at least $2^{m}$ number of $2 m$-tuples in the $2^{2 m}$ number of $2 m$-tuples $\left(\varepsilon_{(q-2) m+1}, \cdots\right.$, $\left.\varepsilon_{q m}\right), \varepsilon_{k}= \pm 1$.

For every $\left(\gamma_{(q-1) m+1}, \cdots, \gamma_{q m}\right) \neq\left(\beta_{(q-1) m+1}, \cdots, \beta_{q m}\right), \gamma_{k}= \pm 1$, we can choose an $m$-tuple $\left(\sigma_{(q-2) m+1}, \cdots, \sigma_{(q-1) m}\right), \sigma_{k}= \pm 1$, such that $\delta^{*}(\omega) \leqq 2^{-m}\left(\omega \in \Omega\left(\sigma_{(q-2) m+1}\right.\right.$, $\left.\left.\cdots, \sigma_{(q-1) m}, \gamma_{(q-1) m+1}, \cdots, \gamma_{q m}, \alpha_{q m+1}, \cdots\right)\right)$. These facts signify that $\delta^{*}(\omega) \leqq 2^{-m}$ $\left(\omega \in \Omega\left(\varepsilon_{(q-2) m+1}, \cdots, \varepsilon_{q m}, \alpha_{q m+1}, \cdots\right)\right)$ holds for the at least $2^{m}+\left(2^{m}-1\right)=$ $2^{2 m}-\left(2^{m}-1\right)^{2}$ number of $2 m$-tuples in the $2^{2 m}$ number of $2 m$-tuples $\left(\varepsilon_{(q-2) m+1}\right.$, $\left.\cdots, \varepsilon_{q m}\right), \varepsilon_{k}= \pm 1$.

Repeating this discussion, we see that $\delta^{*}(\omega) \leqq 2^{-m}\left(\omega \in \Omega\left(\varepsilon_{1}, \cdots, \varepsilon_{q m}, \alpha_{q m+1}, \cdots\right)\right)$ holds for the at least $2^{q m}-\left(2^{m}-1\right)^{q}$ number of $q m$-tuples in the $2^{q m}$ number of $q m$-tuples $\left(\varepsilon_{1}, \cdots, \varepsilon_{q m}\right), \varepsilon_{k}= \pm 1$. 
Since $\operatorname{Pr}\left(\Omega_{0}\right)=1$ and $\left(\alpha_{q m+1}, \alpha_{q m+2}, \cdots\right)$ is arbitrary as long as $\Omega\left(\alpha_{q m+1}, \cdots\right)$ $\neq \Phi$, we have $\operatorname{Pr}\left(\delta^{*}(\omega) \leqq 2^{-m}\right) \geqq 1-\left\{\left(2^{m}-1\right) / 2^{m}\right\}^{q}$. Since $q \geqq 1$ is arbitrary, we have $\delta^{*}(\omega) \leqq 2^{-m}$ a. s.. Since $\operatorname{Pr}\left(\bigcap_{m=1}^{\infty}\left\{\delta^{*}(\omega) \leqq 2^{-m}\right\}\right)=1$, we have $\delta^{*}(\omega)=0$ a. s..

Q.E.D.

3. By the above proposition, it is sufficient to show the existence of such a set and such mappings. To define these, we need the following two lemmas, which are analogous to Lemma 3,6 in [5]. Since these proofs are analogous, we omit the proofs.

LEMMA 1. There exists a constant $C_{0}$ such that, for any sequence $\left(\rho_{n}\right)_{n=1}^{\infty}$, $\rho_{n}>0, \rho_{n} \rightarrow \infty \quad(n \rightarrow \infty)$,

$$
\underset{n \rightarrow \infty}{\limsup } T\left(\rho_{n}, f_{\omega}\right) / T_{0}\left(\rho_{n}\right)>C_{0} \quad \text { a.s.. }
$$

Lemma 2. Put

$$
\left\{\begin{array}{l}
A_{l}(r)=\left(\sum_{k=l}^{\infty}\{k ! /(k-l) !\}^{2}\left|a_{k}\right|^{2} r^{2(k-l)}\right)^{1 / 2} \\
T_{l}(r)=\log ^{+} A_{l}(r) \quad\left(a_{0}=0, l=0,1, \cdots\right) .
\end{array}\right.
$$

Then, for a given $K \geqq 1$, there exists a sequence $\left(r_{n}\right)_{n=1}^{\infty}=\left(r_{n}(K)\right)_{n=1}^{\infty}, r_{n}>0, r_{n} \rightarrow \infty$ $(n \rightarrow \infty)$, such that:

$$
\begin{aligned}
& A_{0}\left(r_{n}+\frac{1}{A_{0}\left(r_{n}\right)}\right) \leqq 2 A_{0}\left(r_{n}\right) . \\
& T_{l}\left(r_{n}+\frac{1}{T_{l}\left(r_{n}\right)}\right) \leqq 2 T_{l}\left(r_{n}\right) \quad(l=0, \cdots, K) .
\end{aligned}
$$

Now we put:

$$
\begin{aligned}
& \Omega_{0}=\bigcap_{m=1}^{\infty} \bigcap_{q=1}^{\infty}\left\{\omega \in \Omega ; \limsup _{n \rightarrow \infty} T\left(r_{n}(m q), f_{\omega}\right) / T_{0}\left(r_{n}(m q)\right)>C_{0}\right\} . \\
& \delta(\omega ; m, q, p)=\liminf _{n \rightarrow \infty, n \in \Delta_{\omega m q}} T\left(r_{n}(m q), 1 / f_{\omega}^{((p-1) m+1)}\right) / T\left(r_{n}(m q), f_{\omega}\right),
\end{aligned}
$$

where $\left(r_{n}(m q)\right)_{n=1}^{\infty}$ is the sequence in Lemma 2 with $K=m q$ and

$$
\Delta_{\omega m q}=\left\{n ; T\left(r_{n}(m q), f_{\omega}\right) / T_{0}\left(r_{n}(m q)\right)>C_{0}\right\} \quad\left(\omega \in \Omega_{0}\right) .
$$

Thus $\Omega_{0}, \delta(\cdot ; m, q, p)(p=1, \cdots, q ; q \geqq 1 ; m \geqq 1)$ are defined.

4. We show that the above $\Omega_{0}, \delta(\cdot ; m, q, p)$ satisfy the conditions in Lemma 1. We see easily $\operatorname{Pr}\left(\Omega_{0}\right)=1$ and (3). For given $m, q \geqq 1$, we must prove that $\delta_{p}(\cdot)=\delta(\cdot ; m, q, p)(p=1, \cdots, q)$ satisfy (4), (5) and (6). So we write simply $r_{n}=r_{n}(m q)(n \geqq 1)$. We see easily (6). To prove (4) and (5), we need 
the following two lemmas. Lemma 3 is proved analogously as in THEOREM 2.1 in [1] and Lemma 4 is analogous to Lemma 8 in [5], and hence we omit the proofs.

LEMMA 3. Let $g(z)$ be an entire function ( $\neq a$ polynomial) and $\left\{P_{j}(z)\right\}_{j=1}^{n}$ multually distinct polynomials of degree $\nu$. Then

$$
\sum_{j=1}^{n} T\left(r, 1 / g_{j}\right) \leqq T\left(r, g^{(\nu+1)}\right)+\sum_{j=1}^{n} \sum_{\mu=1}^{\nu+1} T\left(r, g_{j}^{(\mu)} / g_{j}^{(\mu-1)}\right)+O(\log r),
$$

where $g_{j}(z)=g(z)+P_{\jmath}(z)(\jmath=1, \cdots, n)$.

LEMMA 4. Let $\omega \in \Omega_{0}$. Then, for any $a \in C$ and any $l, 1 \leqq l \leqq q m+1$, we have $T\left(r_{n}, f_{\omega}^{(l)} /\left(f_{\omega}^{(l-1)}-a\right)\right)=o\left(T_{0}\left(r_{n}\right)\right)(n \rightarrow \infty)$.

First we prove (4). Let $\omega \in \Omega_{0}$. For every $a \in \boldsymbol{C}$, we have

$$
\begin{aligned}
& m\left(r_{n}, a, \omega\right)=T\left(r_{n}, 1 /\left(f_{\omega}-a\right)\right) \\
= & T\left(r_{n}, \frac{f_{\omega}^{\prime}}{f_{\omega}-a} \cdot \frac{f_{\omega}^{\prime \prime}}{f_{\omega}^{\prime}} \cdots \frac{f_{\omega}^{((p-1) m+1)}}{f_{\omega}^{((p-1) m)}} \cdot \frac{1}{f_{\omega}^{((p-1) m+1)}}\right) \\
\leqq & T\left(r_{n}, 1 / f_{\omega}^{((p-1) m+1)}\right)+\left\{T\left(r_{n}, f_{\omega}^{\prime} /\left(f_{\omega}-a\right)\right)+\cdots+T\left(r_{n}, f_{\omega}^{((p-1) m+1)} / f_{\omega}^{((p-1) m)}\right)\right\} \\
= & T\left(r_{n}, 1 / f_{\omega}^{((p-1) m+1)}\right)+o\left(T_{0}\left(r_{n}\right)\right),
\end{aligned}
$$

according to Lemma 4 . Hence $\delta(a, \omega) \leqq \delta_{p}(\omega)$. Since this inequality holds for all $a \in \boldsymbol{C}$, we have (4).

Next we prove (5). Let $\omega \in \Omega_{0}$. In the same manner as in (14), we have

$$
T\left(r_{n}, f_{\omega}^{(p m+1)}\right)=T\left(r_{n}, f_{m}\right)+o\left(T_{0}\left(r_{n}\right)\right) \quad(n \rightarrow \infty) .
$$

By Lemma 3, 4 and (15), we have

$$
\begin{aligned}
& \sum_{\omega^{\prime} \in \Gamma(\omega, m, p)} T\left(r_{n}, 1 / \int_{\omega^{\prime}}^{((p-1) m+1)}\right) \\
\leqq & T\left(r_{n}, f_{\omega}^{(p m+1)}\right)+o\left(T_{0}\left(r_{n}\right)\right)=T\left(r_{n}, f_{\omega}\right)+o\left(T_{0}\left(r_{n}\right)\right) \quad(n \rightarrow \infty) .
\end{aligned}
$$

Note that $\lim _{n \rightarrow \infty} T\left(r_{n}, f_{\omega}\right) / T\left(r_{n}, f_{\omega^{\prime}}\right)=1\left(\omega^{\prime} \in \Gamma(\omega, m, p)\right)$ and that there exists $n \geqq 1$ such that $\Delta_{\omega m q} \cap[n,+\infty)=\Delta_{\omega^{\prime} m q} \cap[n,+\infty)$ for all $\omega^{\prime} \in \Gamma(\omega, m, p)$. Divide every term in (16) by $T\left(r_{n}, f_{\omega}\right)$. Letting $n \rightarrow \infty\left(n \in \Delta_{\omega m q}\right)$, we have (5). This completes the proof.

\section{REFERENCES}

[1] Hayman, W.K., Meromorphic functions, Oxford (Clarendon press), 1964.

[2] Kahane, J.P., Some random series of functions, Heath M. M., 1968.

[3] Littlewood, J.E. ANd OfFord, A.C., On the distribution of zeros and $a$-values of a random integral function II, Annals of M. 49 (1948), 885-952.

[4] Murai, T., Une remarque sur la distribution des valeurs des séries de Taylor 
aléatoires, C.R.A.S. Paris, t. 287 (20 nobembre 1978), 931-934.

[5] Murai, T., The value-distribution of random Taylor series in the unit disk, J. London M.S. (3), 24 (1981), 480-494.

[6] Nevanlinna, R., Analytic functions, Springer, 1970.

Department of Mathematics

FACUlTy of ScIENCE

NAgoya University

ChIKUSA-KU, NAgOYA, 464

JAPAN 